\title{
Front Matter: Volume 11447
}

, "Front Matter: Volume 11447," Proc. SPIE 11447, Ground-based and Airborne Instrumentation for Astronomy VIII, 1144701 (4 January 2021); doi: $10.1117 / 12.2591716$

SPIE. Event: SPIE Astronomical Telescopes + Instrumentation, 2020, Online Only 


\section{Ground-based and Airborne Instrumentation for Astronomy VIII}

Christopher J. Evans

Julia J. Bryant

Kentaro Motohara

Editors

14-22 December 2020

Online Only, United States

Sponsored and Published by

SPIE 
The papers in this volume were part of the technical conference cited on the cover and title page. Papers were selected and subject to review by the editors and conference program committee. Some conference presentations may not be available for publication. Additional papers and presentation recordings may be available online in the SPIE Digital Library at SPIEDigitalLibrary.org.

The papers reflect the work and thoughts of the authors and are published herein as submitted. The publisher is not responsible for the validity of the information or for any outcomes resulting from reliance thereon.

Please use the following format to cite material from these proceedings:

Author(s), "Title of Paper," in Ground-based and Airborne Instrumentation for Astronomy VIII, edited by Christopher J. Evans, Julia J. Bryant, Kentaro Motohara, Proceedings of SPIE Vol. 11447 (SPIE, Bellingham, WA, 2020) Seven-digit Article CID Number.

ISSN: 0277-786X

ISSN: 1996-756X (electronic)

ISBN: 9781510636811

ISBN: 9781510636828 (electronic)

Published by

SPIE

P.O. Box 10, Bellingham, Washington 98227-0010 USA

Telephone +1 3606763290 (Pacific Time) · Fax +1 3606471445

SPIE.org

Copyright (c) 2020, Society of Photo-Optical Instrumentation Engineers.

Copying of material in this book for internal or personal use, or for the internal or personal use of specific clients, beyond the fair use provisions granted by the U.S. Copyright Law is authorized by SPIE subject to payment of copying fees. The Transactional Reporting Service base fee for this volume is $\$ 21.00$ per article (or portion thereof), which should be paid directly to the Copyright Clearance Center (CCC), 222 Rosewood Drive, Danvers, MA 01923. Payment may also be made electronically through CCC Online at copyright.com. Other copying for republication, resale, advertising or promotion, or any form of systematic or multiple reproduction of any material in this book is prohibited except with permission in writing from the publisher. The CCC fee code is 0277$786 \mathrm{X} / 20 / \$ 21.00$.

Printed in the United States of America by Curran Associates, Inc., under license from SPIE.

Publication of record for individual papers is online in the SPIE Digital Library.

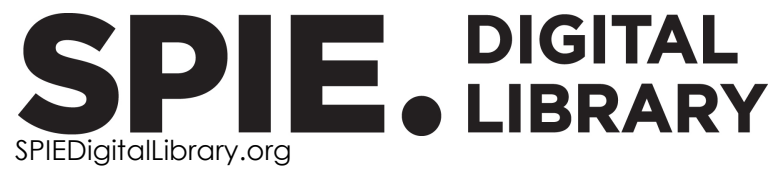

Paper Numbering: Proceedings of SPIE follow an e-First publication model. A unique citation identifier (CID) number is assigned to each article at the time of publication. Utilization of CIDs allows articles to be fully citable as soon as they are published online, and connects the same identifier to all online and print versions of the publication. SPIE uses a seven-digit CID article numbering system structured as follows:

- The first five digits correspond to the SPIE volume number.

- The last two digits indicate publication order within the volume using a Base 36 numbering system employing both numerals and letters. These two-number sets start with 00, 01, 02, 03, 04, $05,06,07,08,09,0 A, 0 B \ldots$ OZ, followed by 10-1Z, 20-2Z, etc. The CID Number appears on each page of the manuscript. 


\section{Contents}

\section{Part One}

MAJOR OBSERVATORIES I

1144702 Instrumentation at the Subaru Selescope [11447-1]

1144706 Current status of the facility instruments at the Large Binocular Telescope Observatory [1 1447-5]

MAJOR OBSERVATORIES II

1144709 Development status of the SOXS spectrograph for the ESO-NTT telescope [11447-8]

11447 OA Status update of LLAMAS: a wide field-of-view visible passband IFU for the $6.5 \mathrm{~m}$ Magellan telescopes [11447-9]

\section{MULTI-MESSENGER INSTRUMENTATION}

$11447 \mathrm{OH} \quad$ Calibration and performance of the readout system based on switched capacitor arrays for the Large-Sized Telescope of the Cherenkov Telescope Array [11447-16]

11447 OJ MeerTRAP in the era of multi-messenger astrophysics [1 1447-18]

NOVEL IMAGERS

11447 OL Rubin commissioning camera: integration, functional testing, and lab performance [1 1447-20]

11447 ON ULTIMATE-Subaru: conceptual design of WFI, a near-infrared wide field imager [11447-22]

1144700 Towards ASTEP+, a two-color photometric telescope at Dome C, Antarctica [1 1447-23]

11447 OP Understanding the Universe for less money: TANGO first robotic ground observatories under extreme conditions [11447-413] 
11447 OT The Planet as Exoplanet Analog Spectrograph (PEAS): design and first-light [11447-153]

SOLAR INSTRUMETATION

11447 OY Sunrise Chromospheric Infrared SpectroPolarimeter (SCIP) for sunrise III: system design and capability [11447-159]

11447 OZ IBIS2.0: The new Interferometric BIdimensional Spectrometer [1 1447-400]

MOS I

1144710 Installation of the Dark Energy Spectroscopic Instrument at the Mayall 4-meter telescope [1 1447-160]

1144711 Performance of the Dark Energy Spectroscopic Instrument (DESI) fiber system [11447-161]

1144714 Integration and early testing of WEAVE: the next-generation spectroscopy facility for the William Herschel Telescope [1 1447-164]

MOS II

$1144715 \quad$ Hector: a new multi-object integral field spectrograph instrument for the Anglo-Australian Telescope [1 1447-234]

$1144716 \quad$ VIRUS2: a next generation replicated integral field spectrograph with wide field and broad wavelength coverage [11447-235]

1144717 Crescent MOONS: an update on the ongoing construction of the new VLT's multi-object spectrograph [11447-236]

1144718 SDSS-V local volume mapper instrument: overview and status [1 1447-237]

MOS III

11447 1A Gemini Infrared Multi-Object Spectrograph: preliminary design overview [1 1447-239]

11447 1B Gemini IRMOS: preliminary optical design of a multi-object adaptive optics-fed infrared integral-field spectrograph [1 1447-240]

11447 ID The Keck-FOBOS spectroscopic facility: conceptual design [11447-242] 
11447 1E The Magellan Infrared Multiobject Spectrograph project [1 1447-243]

HIGH-RESOLUTION SPECTROGRAPHS I

11447 1F On-sky commissioning of MAROON-X: a new precision radial velocity spectrograph for Gemini North [11447-244]

HIGH-RESOLUTION SPECTROGRAPHS II

$114471 \mathrm{~L} \quad$ A microresonator-based etalon for visible light precision radial velocity measurements [1 1447-327]

11447 iN Characterization of a multi-etalon array for ultra-high resolution spectroscopy [11447-328]

HIGH SPATIAL RESOLUTION INSTRUMENTS

11447 1P The Keck Planet Imager and Characterizer: a dedicated single-mode fiber injection unit for high resolution exoplanet spectroscopy [1 1447-330]

11447 IR MAVIS conceptual design [11447-332]

11447 is $\quad$ GPI 2.0: upgrading the Gemini Planet Imager [1 1447-333]

ELT INSTRUMENTATION I

11447 IU A status report on the instruments for ESO's Extremely Large Telescope [11447-335]

11447 IW HARMONI: first light spectroscopy for the ELT: instrument final design and quantitative performance predictions [11447-337]

11447 1Y The Infrared Imaging Spectrograph (IRIS) for TMT: instrument overview [11447-339]

ELT INSTRUMENTATION II

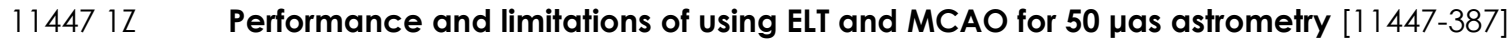




\section{ELT INSTRUMENTATION III}

1144725 MOSAIC: the high multiplex and multi-IFU spectrograph for the ELT [1 1447-393]

1144726 ELT-HIRES, the high resolution spectrograph for the ELT: the Phase A study and the path to construction [11447-394]

1144728 The MANIFEST pre-concept design [11447-396]

POSTER SESSION: ELT INSTRUMENTATION

11447 2D The MICADO first light imager for the ELT: relay optics opto-mechanical design [1 1447-340]

$114472 \mathrm{H} \quad$ HARMONI: first light spectroscopy for the ELT: final design and assembly plan of the spectrographs [11447-343]

$114472 \mathrm{~J}$ Conceptual design of the Giant Magellan Telescope Commissioning Camera [11447-346]

11447 2L HARMONI: first light spectroscopy for the ELT: novel techniques for the calibration of noncommon path aberrations [11447-349]

11447 2N HARMONI: first light spectroscopy for the ELT: simulating the alignment of a three-mirror anastigmat [11447-352]

1144720 The design of the cryostat for ELT/METIS [1 1447-353]

$114472 Q \quad$ Optical interface study of MANIFEST to GMACS and GCLEF instruments [1 1447-357]

11447 2R The warm calibration unit of METIS: laboratory tests and proof-of-concept [1 1447-358]

$114472 T$ Warm calibration unit of the mid-infrared E-ELT instrument METIS: overview and current status of the project [11447-361]

$114472 \mathrm{U} \quad$ An N-band test bench for the METIS coronagraphic masks [11447-362]

11447 2W A mass optimized support and access structure for ESO's Mid-infrared E-ELT Imager and Spectrograph (METIS) [11447-359]

$114472 Z$ The InfraRed Imaging Spectrograph (IRIS) for TMT: photometric characterization of anisoplanatic PSFs and testing of PSF-Reconstruction via AIROPA [1 1447-373]

1144732 HARMONI science path optics: predicting and analysing the expected as-built performance with and end-to-end optical model [11447-370]

1144734 Preliminary design and performance verification of the MICADO Standalone Relay Optics [11447-401] 
1144735 MAORY Instrument Control Hardware: general overview [1 1447-374]

1144737 The Michigan infrared test thermal ELT N-band (MITTEN) cryostat [1 1447-379]

$1144739 \quad \mathrm{CO}_{2}$-based refrigeration system for the NFIRAOS optics enclosure [1 1447-381]

POSTER SESSION: HIGH-RESOLUTION SPECTROGRAPHS

11447 3C The CARMENES M-dwarf planet survey [1 1447-262]

11447 3D Characteristics and performances of an interferometric Doppler imager installed at the $188 \mathrm{~cm}$ telescope of Okayama Observatory [11447-249]

11447 3G ESPRESSO Fiber-Link upgrade. I: Project overview and performances [1 1447-267]

11447 3H Environmental stability achieved for the Manfred Hirth Planet Spectrograph [1 1447-257]

11447 3K MARVEL, a four-telescope array for high-precision radial-velocity monitoring [11447-41]

\section{Part Two}

1144730 A high-resolution echelle spectrograph for precision Doppler observations with small telescopes [11447-316]

$114473 Q \quad$ Fiber-coupling of Fourier transform spectrographs [11447-274]

$114473 R \quad$ Gemini High-Resolution Optical SpecTrograph (GHOST) outer enclosure design and construction [11447-275]

11447 3W NIRPS: integration and validation of thermal performance of the cryogenic near infra-red spectrograph subsystem [11447-309]

$1144740 \quad$ Ghosts of NEID's past [11447-276]

1144741 The NEID port adapter at WIYN: tip-tilt control and vibration analysis [1 1447-277]

$1144742 \quad$ Keck Planet Finder: design updates [11447-278]

1144743 Integration of the Gemini High-Resolution Optical SpecTrograph (GHOST) bench spectrograph [11447-286]

1144744 Stationary Fourier transform spectrometry [11447-250]

1144746 Towards precision radial velocity science with SALT's High-Resolution Spectrograph [11447-287] 
1144747 Bifrost: an ultra-low-cost cross-dispersed optical echelle spectrograph [11447-404]

$1144748 \quad$ Using a passively stabilized Fabry-Perot etalon for determining instrumental artifacts in a spectrograph [1 1447-288]

$114474 \mathrm{H} \quad$ IRANTI: a compact flexible configuration infrared échelle spectrograph integrating emerging technologies for precise radial velocity measurements [11447-261]

$114474 K \quad$ Verification observations of the Manfred Hirt Planet Spectrograph [1 1447-299]

$114474 \mathrm{~L}$ The NEID spectrometer: fibre injection system design [11447-318]

POSTER SESSION: HIGH-SPATIAL RESOLUTION INSTRUMENTS

$114474 R \quad$ The optical alignment of the coronagraphic masks of SHARK-NIR: paving the way for exoplanets detection and characterization [11447-264]

$114474 \mathrm{~T} \quad$ Combination of apodized pupil and phase mask coronagraph for SCExAO at Subaru Telescope [1 1447-254]

$114474 U$ Enhanced high-dispersion coronagraphy with KPIC phase II: design, assembly and status of sub-modules [11447-313]

$114474 X \quad$ Liger for next-generation Keck AO: filter wheel and pupil design [1 1447-280]

$114474 Z$ End-to-end simulation of the SCALES integral field spectrograph [1 1447-294]

1144750 SHARK-NIR: challenges and solutions of a high contrast imager alignment [1 1447-296]

1144752 The segmented pupil experiment for exoplanet detection. 4. A versatile image-based wavefront sensor for active optics [11447-297]

1144753 Laboratory demonstration of focal plane wavefront sensing using phase diversity: a way to tackle the problem of NCPA in SHARK-NIR [1 1447-301]

1144754 An atmospheric dispersion corrector design with milliarcsecond-level precision from 1 to 4 microns for high dispersion coronagraphy [1 1447-302]

$1144755 \quad$ Optical design of a broadband atmospheric dispersion corrector for MAVIS [11447-263]

1144756 The MAVIS Image Simulator: predicting the astrometric performance of MAVIS [11447-265]

$1144758 \quad$ Liger for next-generation Keck adaptive optics: opto-mechanical dewar for imaging camera and slicer [11447-311]

1144759 DiskFM: A forward modeling tool for disk analysis with coronagraphic instruments [11447-251] 
11447 5A Gemini Planet Imager observational calibrations XV: instrument calibrations after six years on sky [1 1447-258]

$114475 B \quad$ Calibration of the instrumental polarization effects of SCExAO-CHARIS' spectropolarimetric mode [1 1447-402]

$114475 D \quad$ GPI 2.0: Upgrades to the IFS including new spectral modes [11447-410]

POSTER SESSION: MAJOR OBSERVATORIES

11447 5E WALOP-South: A wide-field one-shot linear optical polarimeter for PASIPHAE survey [1 1447-24]

11447 5F SOXS: effects on optical performances due to gravity flexures, temperature variations, and subsystems alignment [11447-70]

$114475 \mathrm{H} \quad$ The University of Tokyo Atacama Observatory 6.5m telescope: On-sky performance of the near-infrared instrument SWIMS on the Subaru telescope [11447-33]

$1144751 \quad$ Conceptual design of a high-resolution ultra-stable spectrograph for GTC [1 1447-74]

11447 5J Cerberus: A three-headed instrument for the OARPAF telescope [11447-88]

11447 5K MuSCAT3: a 4-color simultaneous camera for the 2m Faulkes Telescope North [1 1447-29]

$114475 \mathrm{~L} \quad$ Progress on the UV-VIS arm of SOXS [11447-36]

$114475 \mathrm{M} \quad$ Curved detector-based optical design for the VLT/BlueMUSE instrument [1 1447-77]

$114475 \mathrm{~N} \quad$ The development status of the NIR Arm of the new SoXS instrument at the ESO/NTT telescope [1 1447-90]

1144750 The current status of the imaging and spectrogragh (IMSP) for $4 \mathrm{~m}$ telescope [11447-30]

11447 5P Gemini infrared multi-object spectrograph: calibration system [11447-78]

$114475 R \quad$ Final design and performance of the Fully Automatic Spectrograph for Transient (FAST) [11447-106]

11447 5T Performances of an integral field unit for FOCAS on the Subaru telescope [1 1447-35]

$114475 \mathrm{U} \quad$ Further development and testing of TCal: a mobile spectrophotometric calibration unit for astronomical imaging systems [1 1447-108]

$114475 \mathrm{~V} \quad$ Final design and development status of the acquisition and guiding system for SOXS [11447-80]

11447 5W A simple concept for atmospheric dispersion correctors on-sky commissioning tests [11447-102] 
$114475 X \quad$ The University of Tokyo Atacama Observatory 6.5m telescope: On-sky performance evaluations of the mid-infrared instrument MIMIZUKU on the Subaru telescope [1 1447-38]

$114475 Z$ Multi-wavelength SimultaneouS High throughput Imager and polarimeter (MuSaSHI): development and its performance [1 1447-42]

1144760 Stellar astrophysics in the near-UV with VLT-CUBES [11447-104]

$1144763 \quad$ Multifocal station for the Wendelstein 2m Fraunhofer Telescope [1 1447-43]

$1144764 \quad$ Update on the preliminary design of SCALES: the Santa Cruz Array of Lenslets for Exoplanet Spectroscopy [11447-110]

1144765 Optical aspects of Near-Infrared Imager Spectrometer and Polarimeter instrument (NISP) [11447-46]

1144766 Design and development of the SOXS calibration unit [1 1447-45]

1144767 Design requirements for the Wide-field Infrared Transient Explorer (WINTER) [11447-113]

1144768 A dual-beam spectrograph design for transient follow-up with SALT [11447-47]

1144769 Development of the ROSIE integral field unit on the Magellan IMACS spectrograph [1 1447-116]

11447 6A Flexure updates to MOSFIRE on the Keck I telescope [11447-114]

$114476 C$ Development status of the UV-VIS detector system of SOXS for the ESO-NTT telescope [11447-52]

11447 6E Stray light analysis and reduction for IFU spectrograph LLAMAS [11447-118]

$114476 \mathrm{H} \quad$ Ground support facilities of the WSO-UV space mission [11447-119]

$1144761 \quad$ The University of Tokyo Atacama Observatory 6.5m telescope: update of the Near-Infrared Echelle Spectrograph NICE as a first light instrument [1 1447-54]

$114476 \mathrm{~K}$ On-sky examination of optical diffusers installed in MuSCAT [11447-58]

$114476 \mathrm{~L}$ TSPEC4: near-IR spectroscopy for the SOAR telescope [11447-120]

$114476 \mathrm{~N} \quad$ New technologies for the Tenerife Microwave Spectrometer and current status [11447-63]

$114476 \mathrm{P} \quad$ The AIV strategy of the common path of Son Of X-Shooter [11447-60]

$114476 Q \quad$ Preliminary mechanical design of the Gemini Infrared Multi-Object Spectrograph (GIRMOS) Cryostat [11447-123] 
$114476 R \quad$ A novel method for on-sky measurements of atmospheric dispersion [1 1447-64]

\section{Part Three}

$114476 \mathrm{U} \quad$ Mechanical aspects of near infrared imager spectrometer and polarimeter [11447-62]

$114476 Z$ GATOS: A fast multi-channel imager and spectrograph for the Gran Telescopio Canarias [11447-71]

1144771 SCALA upgrade: development of a light source for sub-percent calibration uncertainties [1 1447-72]

1144774 SCORPIO: Final design and performance estimates for time-domain astronomy [11447-144]

1144776 Conceptual design of the BRONCO spectrograph [1 1447-86]

$1144778 \quad$ Electronics design and development of near-infrared imager, spectrometer, and polarimeter [1 1447-89]

11447 7A FORS-Up: Making the most versatile instrument in Paranal ready for 15 more years of operations [1 1447-97]

11447 7C Operational modes and efficiency of SOXS [11447-99]

11447 7D The Exoplanet Transmission Spectroscopy Imager (ETSI) [1 1447-100]

$114477 \mathrm{E} \quad$ Ongoing and future instrument upgrades at Gemini [11447-101]

11447 7F Developing Modular Adaptive Transition Edge Sensor SQUID Electronics (MATESSE) [1 1447-141]

11447 7G Panoramic SETI: overall focal plane electronics and timing and network protocols [11447-149]

\section{POSTER SESSION: MULTI-OBJECT SPECTROGRAPHS}

11447 7R Final assembly, metrology, and testing of the WEAVE fibre positioner [1 1447-165]

11447 7V Prime Focus Spectrograph (PFS): the prime focus instrument [11447-175]

11447 7W MOONS-ESO spectrograph: status of the cryogenic opto-mechanical system for movable optics [11447-168]

$114477 Y$ Starbugs field-allocation simulations for FOBOS [11447-169] 
$1144781 \quad$ A robotic Focal Plane System (FPS) for the Sloan Digital Sky Survey V [1 1447-173]

1144783 DMD based flat field calibration system for MOONS [1 1447-185]

$1144784 \quad$ Prime Focus Spectrograph (PFS): the metrology camera system [11447-174]

1144785 The DESI sky continuum monitor system [1 1447-187]

1144786 Testing the 10 spectrograph units for DESI: approach and results [11447-179]

1144787 4MOST low resolution spectrograph MAIT [1 1447-188]

11447 8B Mauna Kea Spectrographic Explorer (MSE): new preliminary design for the multi-object high resolution spectrograph [11447-191]

11447 8D MOONS, the next ESO VLT's multi-object spectrograph: the field corrector and the rotating front end [11447-184]

$114478 G \quad$ The Hector Instrument: performance of the Hector fibre integral field units [11447-189]

11447 8K Performance of the Dark Energy Spectroscopic Instrument (DESI) focal plane [11447-193]

$114478 \mathrm{~N} \quad$ The SDSS-V local volume mapper fiber cable system [1 1447-204]

1144780 SDSS-V focal plane robot positioning metrology [11447-215]

$114478 \mathrm{P} \quad$ Test results of the SDSS-V fiber micro-positioners [1 1447-217]

$114478 Q \quad$ The 4MOST secondary guider imaging system [11447-210]

$114478 \mathrm{~T} \quad$ Testing fiber tapers for use in the SDSS-V focal plane system [1 1447-206]

$114478 \mathrm{U} \quad$ The Hector Instrument: optical design of the new higher-resolution spectrograph [11447-219]

$114478 \mathrm{~W} \quad$ Mechanical design of the VIRUS2 instrument [11447-221]

$114478 Y \quad$ The prototype telescope and spectrograph system for the AMASE project [1 1447-225]

$114478 Z \quad$ VIRUS2: Interfaces to the 2.7 m Harlan J Smith Telescope [1 1447-226]

1144790 Design of a theta/phi fiber positioner robot for the Sloan Digital Sky Survey V [1 1447-227]

$1144791 \quad$ Bending losses in multi-mode fibers with low numerical aperture [1 1447-229] 
1144794 Performance of Kitt Peak's Mayall 4-meter telescope during DESI commissioning [1 1447-399]

$1144795 \quad$ Maunakea Spectroscopic Explorer Low Moderate Resolution Spectrograph: paths toward the Preliminary Design Phase [1 1447-407]

POSTER SESSION: NOVEL IMAGERS

$1144796 \quad$ Microbolometer arrays for ground-based infrared imaging [11447-27]

1144798 Detection and calculation of meteor trajectories by MOFID All Sky cameras network [11447-105]

11447 9A Fabrication and characterization of filters for the Large Synoptic Survey Telescope [11447-39]

11447 9B The PICTURE-C MKID camera [11447-79]

11447 9E EMPOL: an EMCCD based optical imaging polarimeter [1 1447-44]

$114479 F \quad$ Opto-mechanical design of the Wide-Field Infrared Transient Explorer (WINTER) fly's eye camera [11447-115]

$1144791 \quad$ Performance of the BSTI instrument on the NUTTelA-TAO telescope for high time-resolution, simultaneous three-channel imaging of prompt gamma-ray burst optical emission [1 1447-50]

11447 9K The wide-field infrared transient explorer (WINTER) [11447-55]

11447 9M Development of flat fielding method in MIR for accurate photometry with TAO/MIMIZUKU [1 1447-59]

$114479 U$ (KFISP) Kottamia Faint Imaging Spectro-Polarimeter: opto-mechanical design and performance analysis [1 1447-75]

11447 9W (KFISP) Kottamia Faint Imaging Spectro-Polarimeter: software, motion control, performance and results [11447-76]

$114479 Y \quad$ A coronagraph using a digital micromirror device as an adaptive occultation mask: design and observational result [1 1447-284]

$1144797 \quad$ Using the OWL@OUKA telescope to follow-up the TESS planet candidates: first results [11447-93]

11447 A0 MAVIS: science case, imager, and spectrograph [11447-285]

11447 A $1 \quad$ New narrow-band filter system for the IRSF 1.4m telescope [11447-405]

11447 A2 A heterogeneous telescope array optimized for low surface-brightness imaging [11447-409] 
POSTER SESSION: SOLAR

11447 A3 Sunrise Chromospheric Infrared spectroPolarimeter (SCIP) for SUNRISE III: polarization modulation unit [11447-166]

11447 A5 Development of new tunable filter for solar observation in Hida observatory [11447-176]

11447 A9 Observing the integrated and spatially resolved Sun with ultra-high spectral resolution [11447-156]

11447 AB Sunrise Chromospheric Infrared spectroPolarimeter (SCIP) for SUNRISE III: opto-mechanical analysis and design [11447-195]

$11447 \mathrm{AF} \quad$ Design of an integral field unit for a solar telescope [11447-203]

11447 AG CYRA: the cryogenic infrared spectrograph for the Goode Solar Telescope in Big Bear [11447-213]

11447 AJ Sunrise Chromospheric Infrared spectroPolarimeter (SCIP) for SUNRISE III: optical design and performance [11447-205]

11447 AK The SUNRISE UV Spectropolarimeter and imager for SUNRISE III [1 1447-220]

11447 AM Polarimeter for the HELLRIDE instrument at Vacuum Tower Telescope [11447-231] 